\title{
Under-the-barrier dynamics in laser-induced relativistic tunneling
}

\author{
Michael Klaiber, Enderalp Yakaboylu, Heiko Bauke, Karen Z. Hatsagortsyan, and Christoph H. Keitel \\ Max-Planck-Institut für Kernphysik, Saupfercheckweg 1, 69117 Heidelberg, Germany
}

(Dated: November 1, 2018)

\begin{abstract}
The tunneling dynamics in relativistic strong-field ionization is investigated with the aim to develop an intuitive picture for the relativistic tunneling regime. We demonstrate that the tunneling picture applies also in the relativistic regime by introducing position dependent energy levels. The quantum dynamics in the classically forbidden region features two time scales, the typical time that characterizes the probability density's decay of the ionizing electron under the barrier (Keldysh time) and the time interval which the electron spends inside the barrier (Eisenbud-Wigner-Smith tunneling time). In the relativistic regime, an electron momentum shift as well as a spatial shift along the laser propagation direction arise during the under-the-barrier motion which are caused by the laser magnetic field induced Lorentz force. The momentum shift is proportional to the Keldysh time, while the wave-packet's spatial drift is proportional to the Eisenbud-Wigner-Smith time. The signature of the momentum shift is shown to be present in the ionization spectrum at the detector and, therefore, observable experimentally. In contrast, the signature of the Eisenbud-Wigner-Smith time delay disappears at far distances for pure quasistatic tunneling dynamics.
\end{abstract}

PACS numbers: 32.80.Rm, 31.30.J-, 03.65.Xp

Introduction With present-day strong lasers providing intensities of the order of $10^{22} \mathrm{~W} / \mathrm{cm}^{2}$ [1] and employing highly charged ions, the relativistic regime of strong field atomic processes, in particular, strong field ionization of highly charged ions [2], is accessible [3]. Relativistic generalizations of the strong field approximation (SFA) [4-7] and the quasiclassical theory of ionization [8, 9] provide simple ways to calculate ionization probabilities in the relativistic regime [10, 11].

The ionization occurs in the so-called tunneling regime when the laser's electric field amplitude $E_{0}$ is smaller than those required for over-the-barrier ionization [12] and the Keldysh parameter $\gamma=\omega \sqrt{2 I_{p}} / E_{0}$ [13] is well below unity with the ionization potential $I_{p}$ and the laser's angular frequency $\omega$. The Keldysh parameter defines the time scale $\tau_{K}=\gamma / \omega$ during which individual momentum components of the ionized wave packet are formed [5]. When this time scale is shorter than the laser period, i. e., $\gamma \ll 1$, the laser field can be treated as quasistatic.

In the nonrelativistic as well as in the relativistic case, the electron travels during its journey from the bound state into the continuum through a region which is forbidden according to classical mechanics. For nonrelativistic dynamics, when the typical electron velocities are negligible compared to the speed of light, it is legitimate to treat the laser field as a homogeneous time-dependent electric field $\boldsymbol{E}(t)$ and to neglect its magnetic component. In this case a well-known intuitive picture for the tunneling regime arises in which the electron tunnels out through the effective potential $V_{\text {eff }}(\boldsymbol{r}, t)=V(\boldsymbol{r})+\boldsymbol{r} \cdot \boldsymbol{E}(t)$ formed by the atomic potential $V(\boldsymbol{r})$ and the laser field. The Keldysh time $\tau_{K}$ may be interpreted as the time that a classical free electron would need to cover the length $l \sim I_{p} / E_{0}$ of the tunneling barrier at the characteristic velocity of a bound electron $v_{a}=\sqrt{2 I_{p}}$. The contribution of the laser's magnetic field component becomes non-negligible when we enter the relativistic regime. A transverse laser field with perpendicular electric and magnetic components cannot be described solely by a scalar potential. Consequently, a generalization of the tunneling picture into the relativistic regime is not straightforward [14] and there is no clear intuitive picture for the relativistic quasistatic ionization dynamics in the classically forbidden region.

Later, we will identify the so-called tunneling time $\tau_{E}$ as a second time scale that is fundamental for the electron dynamics in the classically forbidden region. The tunneling time through a potential barrier (Eisenbud-Wigner-Smith time delay [15]) has been a long-standing problem in quantum mechanics [16[18]. Interest to this problem has been renewed recently by experimental attempts to measure the temporal delay at the electron emergence into the continuum from a bound state during the laser-induced (nonrelativistic) tunneling [19]. A vanishing tunneling time within the experimental error has been observed in these experiments.

In order to develop an intuitive picture for the relativistic tunneling regime of strong field ionization, we carry out a detailed analysis of the electron dynamics through the classically forbidden region in the relativistic regime and compare it with the corresponding nonrelativistic case. The characteristic time scales are examined. Our analysis is based on the SFA [4-7] as well as on the exact solution of the relativistic wave equations and numerical simulations. The possibility for the experimental detection of the specific relativistic features of the classically forbidden dynamics is also elaborated.

System A highly charged hydrogenlike ion with nuclear charge $Z \lesssim 100$ at the origin of our coordinate system is considered to interact with a strong linearly polarized laser field with electric field of amplitude $E_{0}$ in $x$-, magnetic field of amplitude $B_{0}$ in $y$-and propagation in $z$-direction $\left(E_{0}=B_{0}\right)$. Position and momenta of electrons that are ionized by the laser pulse may be measured by a detector far away from the interaction zone. Throughout this Letter we describe the laser field via its vector potential in electric field gauge [20, 21], i. e., $\phi(\boldsymbol{r}, t)=-\boldsymbol{r} \cdot \boldsymbol{E}(\eta)$ and $\boldsymbol{A}(\boldsymbol{r}, t)=-\hat{\boldsymbol{k}}[\boldsymbol{r} \cdot \boldsymbol{E}(\eta)] / c$ with the laser phase $\eta=t-z / c$, the electron's coordinate $\boldsymbol{r}=(x, y, z)$, the 
unit vector in laser propagation direction $\hat{\boldsymbol{k}}$, and the speed of light $c$. The dynamics of the electron state $|\Psi\rangle$ in the rest frame of the ionic core is then defined via the Dirac equation

$$
\mathrm{i} \partial_{t}|\Psi\rangle=\left\{c \boldsymbol{\alpha} \cdot[\hat{\boldsymbol{p}}+\boldsymbol{A}(\boldsymbol{r}, t)]+V(\boldsymbol{r})-\phi(\boldsymbol{r}, t)+\beta c^{2}\right\}|\Psi\rangle
$$

with the momentum operator $\hat{\boldsymbol{p}}$ and the Dirac matrices $\boldsymbol{\alpha}$ and $\beta$. In the following, we use an extreme but feasible set of parameters, viz. $I_{p} / c^{2}=0.25$ and $E_{0} / E_{a}=1 / 30$, with $E_{a}=Z^{3}$ denoting the characteristic atomic field strength, representing a hydrogenlike ion with nuclear charge of $Z \approx 90$ in the tunneling regime [12].

Relativistic strong field approximation The SFA allows us to calculate the ionized part of the asymptotic electron wave packet at a detector in the remote future. As we are interested in the under-the-barrier dynamics of the electron, the momentum distribution at the tunnel exit time $t_{0}$ is deduced analytically from the distribution at the detector, assuming free propagation in the laser field outside the barrier. The asymptotic ionization amplitude in momentum space reads [22]

$$
\left\langle\boldsymbol{p}_{f}, s_{f} \mid \Psi\right\rangle=\int_{-\infty}^{\infty}\left\langle\psi_{\boldsymbol{p}_{f}, s_{f}}|\boldsymbol{r} \cdot \boldsymbol{E}(\eta)| \tilde{\psi}_{0}\right\rangle \mathrm{d} \eta,
$$

where $\boldsymbol{p}_{f}=\left(p_{f, x}, p_{f, y}, p_{f, z}\right)$ and $s_{f}$ denote the electron's final momentum and spin, respectively, $\left|\psi_{p, s}\right\rangle$ is the Volkov state [23] with momentum $\boldsymbol{p}$ and spin $s$, while $\left|\tilde{\psi}_{0}\right\rangle$ is the dressed Dirac bound state spinor of a short range potential with binding energy $-I_{p}[22]$. The relativistic wave function $\left|\psi_{0}\right\rangle$ is used in the relativistic as well as in the nonrelativistic calculation allowing to trace back the features in the under-the-barrier dynamics to the Hamiltonian's different corrections to the electric dipole approximation. Calculating the integral (2) via the saddle point method, the momentum distributions at the detector, see Fig.11(a), and at the tunnel exit are derived; see Fig. 1(b). While parabolic wings of size $p_{f, z} \sim E_{0}^{2} /\left(\omega^{2} c\right)$ are a common feature of asymptotic momentum distributions for ionization in the relativistic regime [22], Fig. 11 (a) reveals also a shift of the global maximum of the asymptotic momentum distribution from $\left(p_{f, x}, p_{f, z}\right)=(0,0)$ in the nonrelativistic regime to $\left(0, I_{p} /(3 c)\right)$ in the relativistic regime [24]. The shift of the global maximum arises during the under-the-barrier motion. This may be realized by calculating the distribution of the kinetic momentum at the exit nonrelativistically and comparing it with the fully relativistic result; see Fig. 11(b). To identify the relevant relativistic effects, we expand the Dirac equation (1) retaining the magnetic dipole correction and the leading relativistic correction to the electron's kinetic energy (termed mass-shift correction)

$$
\begin{aligned}
& \mathrm{i} \partial_{t}|\Psi\rangle= \\
& \left\{\frac{\hat{p}_{x}^{2}}{2}+\frac{\hat{p}_{y}^{2}}{2}+\frac{\left[\hat{p}_{z}-\boldsymbol{r} \cdot \boldsymbol{E}(t) / c\right]^{2}}{2}-\frac{\hat{\boldsymbol{p}}^{4}}{8 c^{2}}+V(\boldsymbol{r})+\boldsymbol{r} \cdot \boldsymbol{E}(t)\right\}|\Psi\rangle .
\end{aligned}
$$

This expansion is justified because relativistic effects for the under-the-barrier motion are determined by the parameter $I_{p} / c^{2}$ and even for the extreme parameter set used the tunneling process is only weakly relativistic. This can be seen from Fig. 1(b), where the calculation based on Eq. (3) recovers the exact result from Eq. (1). Furthermore, the figure shows that the magnetic dipole term (Lorentz-force) is responsible for the momentum shift and a decrease of the tunneling probability whereas the mass-shift correction counteracts the latter effect.

A quasiclassical picture for relativistic tunneling For a qualitative interpretation of the tunneling procedure $(\gamma \ll 1)$ and the momentum shift, we omit the mass-shift correction as justified above and employ the quasistatic approximation in the quasiclassical picture. Then the Hamilton function corresponding to Eq. (3) yields at maximal laser intensity

$$
H=\frac{p_{x}^{2}}{2}+\frac{p_{y}^{2}}{2}+\frac{\left(p_{z}+x E_{0} / c\right)^{2}}{2}+V(r)-x E_{0},
$$

with the canonical momenta $p_{x}, p_{y}$, and $p_{z}, r=\sqrt{x^{2}+y^{2}+z^{2}}$. In (4) the electric field gauge [20, 21] ensures that the Hamilton function equals the total energy $-I_{p}$. Similarly as shown in the nonrelativistic case [12], we estimated numerically that the relativistic deviations still result in tunneling dynamics in close vicinity along the $x$-axis (the polarization direction). The atomic potential's central force in the direction of the $y$-axis and the $z$-axis (the laser's propagation directions) is then also negligible in the tunneling region and the canonical momenta $p_{y}$ and $p_{z}$ become approximately conserved. Consequently, the electron dynamics is separable and the equation $H=-I_{p}$ can be written as

$$
-I_{p}-\left[\frac{p_{y}^{2}}{2}+\frac{\left(p_{z}+x E_{0} / c\right)^{2}}{2}\right]=\frac{p_{x}^{2}}{2}+\left[V(x)-x E_{0}\right] .
$$

Equation (5) is a generalization of an often applied nonrelativistic one-dimensional model [12]. It may be interpreted as
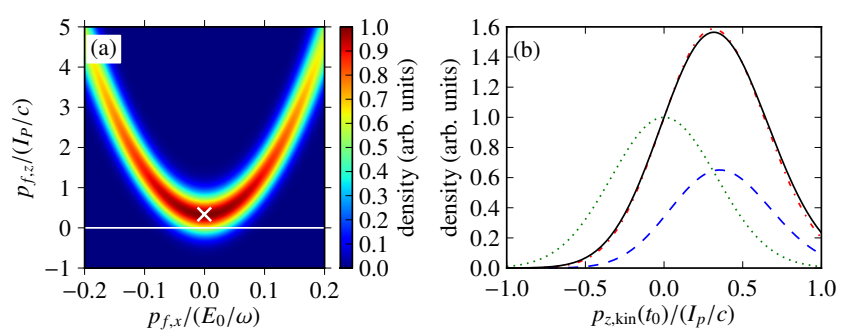

FIG. 1: (a) Density plot of the asymptotic spin-averaged electron momentum distribution at the detector. The distribution's maximum (white cross) is shifted from $p_{f, z}=0$ (nonrelativistic limit, white line) to $p_{f, z}=I_{p} /(3 c)$. (b) The distribution of the electron's kinetic momentum in the laser propagation direction at the tunnel exit at time $t_{0}$, with maximal laser electric field, in the electric dipole approximation (green, dotted line), including the magnetic dipole correction (blue, dashed line) and the magnetic dipole plus the mass-shift correction (red, dot-dashed line) and fully relativistically via the Dirac equation in the SFA (black, solid line). The applied parameters are $I_{p} / c^{2}=0.25$ and $E_{0} / E_{a}=1 / 30$. 


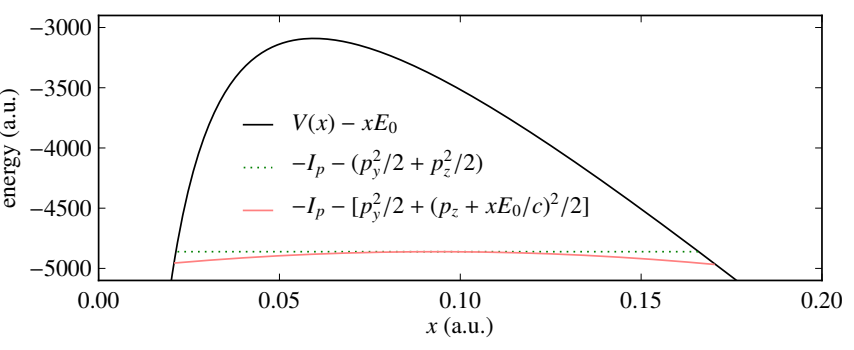

FIG. 2: (color online) Scheme of laser induced tunneling in a Coulomb potential with $I_{p} / c^{2}=0.25$. The potential barrier $V(x)-x E_{0}$ (black line) is displayed with $E_{0} / E_{a}=1 / 30$ and the relativistic and nonrelativistic modified energy levels. The dotted green line indicates the description in electric dipole approximation, whereas magnetic dipole effects are taken into account for the solid red line. The momenta $p_{y}$ and $p_{z}$ are chosen such that the WKB tunneling probability is maximal.

follows. During the quasi one-dimensional motion along the laser polarization direction, the electron propagates through the barrier $V_{\text {eff }}(x)=V(x)-x E_{0}$ with a position dependent total energy $\varepsilon(x)=-I_{p}-\left\{p_{y}^{2} / 2+\left[p_{z}+x E_{0} / c\right]^{2} / 2\right\}$, which is the difference between the binding energy and the kinetic energy of the motion in the propagation direction of the laser. Equation (5) yields the momentum in electric field direction $p_{x}=\mathrm{i} \sqrt{2\left[V_{\text {eff }}(x)-\varepsilon(x)\right]}$. Note, that the kinetic momentum in the propagation direction of the laser $p_{z, \text { kin }}(x)=p_{z}+x E_{0} / c$ in the laser's propagation direction is shifted due to the Lorentz force during the under-the-barrier motion causing a modification of $\varepsilon(x)$ and, consequently, a modification of the momentum $p_{x}$ as compared to the nonrelativistic case where the kinetic momentum $p_{z, \text { kin }}(x)=p_{z}$ is conserved. The WKB tunneling probability is proportional to $\exp (-\Gamma)$, where $\Gamma$ is given by the integral $\Gamma=-2 \mathrm{i} \int_{x}^{x_{e}} p_{x} \mathrm{~d} x$ over the classical forbidden region $x_{i} \leq x \leq x_{e}$ [8, 11]. For short range potentials the WKB tunneling probability is maximal when tunneling starts with a negative kinetic momentum $p_{z, \mathrm{kin}}\left(x_{i}\right)=-2 I_{p} /(3 c)$ reaching the tunnel exit with positive $p_{z, \text { kin }}\left(x_{e}\right)=I_{p} /(3 c)$. At this optimal momentum the energy $\varepsilon(x)$ is strictly below $-I_{p}$, which is the corresponding expression in the case of the electric dipole approximation, see Fig. 2. This indicates a decrease of the maximal tunnel probability with respect to the nonrelativistic case, cf. with Fig.11(b).

In the WKB approximation the electron wave function evolves along quasiclassical trajectories [8, 11]. Under the barrier, they are described by classical Hamiltonian mechanics developing in imaginary time. The imaginary time determines the exponential damping of the ionized electron wave function during tunneling. In particular, a time interval $-\mathrm{i} \tau_{K}$ is required to pass the barrier; thus, the wave function is reduced by a factor $\mathrm{e}^{-I_{p} \tau_{K}}$ while tunneling. Under the barrier the Lorentz force of order $v_{a} B_{0} / c$ acting for the Keldysh time $\tau_{K}$ causes a total momentum shift of $\Delta p_{z, \text { kin }}=p_{z, \text { kin }}\left(x_{e}\right)-p_{z, \text { kin }}\left(x_{i}\right) \sim$ $\tau_{K} v_{a} B_{0} / c \sim\left(x_{e}-x_{i}\right) E_{0} / c \sim I_{p} / c$. In order to compensate for the acceleration in positive $z$-direction by the Lorentz force, the
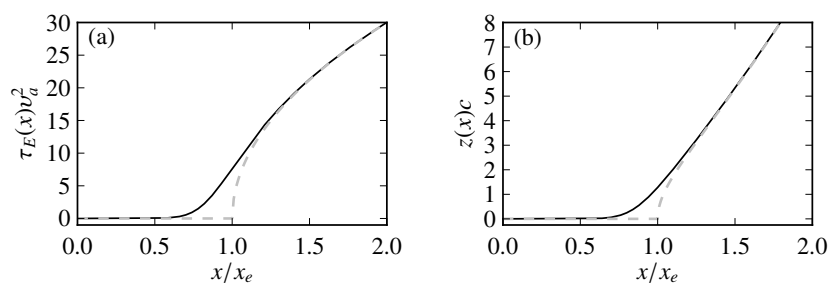

FIG. 3: (a) The scaled time delay vs. the coordinate along the laser polarization direction, via a quantum mechanical (black, solid line) and a quasiclassical description (gray, dashed line). (b) The electron wave packet trajectory in the $x$-z-plane, via a quantum mechanical (black, solid line) and a quasiclassical description (gray, dashed line). In both subfigures $x / x_{e}<1$ corresponds to under-the-barrier motion with $x_{e}=I_{p} / E_{0}$ denoting the tunnel exit coordinate. The applied parameters are $I_{p} / c^{2}=0.25, E_{0} / E_{a}=1 / 30$, and the most probable initial kinetic momenta $p_{z, \text { kin }}\left(x_{i}\right)=-2 I_{p} /(3 c)$ and $p_{y}=0$.

$z$-component of the initial kinetic momentum at entering the barrier has to be negative similar to [25]. The kinetic momentum in the propagation direction of the laser is positive at the barrier exit, see the dashed blue lines in Fig.11(b), in contrast to the nonrelativistic case where it vanishes. Considering the mass-shift correction in addition to the magnetic dipole terms increases the tunneling probability; see Fig. 1(b). The general tunneling picture, however, is not affected.

Tunneling time We point out that although the Lorentz force induces a momentum shift along the laser propagation direction during the under-the-barrier motion, nevertheless, in the quasistatic WKB approximation the tunneling process happens instantaneously and thus there is no real coordinate drift in laser propagation direction as a result of the under-the-barrier motion. However, in reality the under-the-barrier motion requires a small nonzero time span which can be derived by going beyond the quasistatic WKB theory.

For this purpose we use the exact wave function of the electron in the quasistatic potential formed by the laser field and a zero-range atomic potential. The kinetic energy term of the Schrödinger equation with the magnetic dipole corrections is expanded in the coordinate $x$ around the tunnel exit coordinate $x_{e}$. Then, the electron wave function in the continuum is derived as

$$
\psi_{+}(x)=T \operatorname{Ai}\left\{\mathrm{e}^{-\mathrm{i} \pi / 3}\left[2^{1 / 3}\left(x E_{0}-I_{p}\right) / E_{0}^{2 / 3}+\phi(x)\right]\right\},
$$

where $\phi(x)=\left(p_{z} c+I_{p}\right)\left(3 p_{z} c+2 x E_{0}+I_{p}\right) / 3 c^{2}\left(2 E_{0}\right)^{2 / 3}$, and $\mathrm{Ai}$ denotes the Airy function. The real transmission coefficient $T$ is obtained by matching $\psi_{+}(x)$ with the bound state wave function [11] $\psi_{0}(x)=$ $\sqrt{2 \pi v_{a}} /\left[v_{a}+\left(p_{y}^{2}+p_{z}^{2}\right) /\left(2 v_{a}\right)\right] \exp \left\{-\left(v_{a}+\left(p_{y}^{2}+p_{z}^{2}\right) /\left(2 v_{a}\right)\right] x\right\}$.

The time it takes for the maximum of the wave packet to reach position $x$ [Eisenbud-Wigner-Smith time delay $\tau_{E}(x)$ ] and the drift of the electron wave packet in propagation direction at position $x$ during the under-the-barrier motion are determined by the phase of the wave function $\arg \left[\psi_{+}(x)\right]$ [17], viz. $\tau_{E}(x)=-\partial \arg \left[\psi_{+}(x)\right] / \partial I_{p}$ and $z(x)=-\partial \arg \left[\psi_{+}(x)\right] / \partial p_{z}$. Figure 3 (a) shows that the electron spends some time un- 
der the barrier when it is close to the exit. The tunneling time, i.e., the time delay at the tunneling exit is given by $\tau_{E}\left(x_{e}\right) \approx 3^{5 / 6} \sqrt{\pi}\left(E_{a} / E_{0}\right)^{2 / 3} /\left[2 \Gamma(1 / 6) I_{p}\right]$ and the drift distance at the tunneling exit is $z\left(x_{e}\right) \approx \sqrt{\pi} I_{p} /\left[3^{1 / 6} \Gamma(1 / 6) E_{0}^{2 / 3} c\right]$. The tunneling time of order $1 / I_{p}$ may also be estimated via Heisenberg's uncertainty relation when assuming an energy uncertainty of order of $I_{p}$. The drift size along the laser propagation direction at the tunneling exit is proportional to the tunneling time with a proportionality factor that is equal to the kinetic momentum in the laser's propagation direction at the tunnel exit, $z\left(x_{e}\right)=p_{z \text {,kin }}\left(x_{e}\right) \tau_{E}\left(x_{e}\right)$. This indicates that the drift is shaped during the Eisenbud-Wigner-Smith tunneling time. Note that for the applied parameters the drift size at the tunneling exit $z\left(x_{e}\right)$ is of order of $1 / c$. The drift along the laser propagation direction during the under-the-barrier motion is due to the Lorentz force and disappears in the nonrelativistic limit. The time delay $\tau_{E}(x)$, however, behaves similarly to that shown in Fig. 3 (a) also in the nonrelativistic regime.

The time delay far from the tunnel exit, that means $x-x_{e} \gtrsim$ $E_{0}^{-1 / 3}$, follows approximately $\left.\tau_{E}(x)\right|_{x \rightarrow \infty} \approx \sqrt{2\left(x-x_{e}\right) / E_{0}}$. The latter corresponds to the quasiclassical motion time of the electron which tunnels instantaneously and continues at $x_{e}$ with vanishing initial momentum in the laser's electric field direction $p_{x}=0$, reaching $x$ at a moment $t\left(x-x_{e}=E_{0} t^{2} / 2\right)$. Therefore, we can conclude that the signature of the nonzero tunneling time exists only in a small area near the tunnel exit at distances $\delta x \sim E_{0}^{-1 / 3}$ and disappears at far distances near the detector, see Fig. 3 (b). This consequence is relevant also in the nonrelativistic case and explains the observation of the vanishing tunneling time in the experiments [19]. Mathematically, the problem of the vanishing Wigner time at large distances arises when the laser-induced quasistatic barrier is linear in the $\mathrm{x}$ coordinate around the exit of the tunneling electron in the interval $\left[x_{e}-\delta x, x_{e}+\delta x\right]$, which is the case when the saddle point of the effective potential barrier formed by the laser and atomic potentials is far from the tunneling exit. Instead, in the regime close to over-the-barrier ionization at $E_{0} / E_{a}>1 / 25$ the effective potential has a parabolic character and analog calculations as carried out here indicate a measurable Eisenbud-WignerSmith tunneling time [26]. In interpreting experimental results on this tunneling time, the distortion of the electron trajectories by the Coulomb field of the ionic core during the sub-barrier as well as out-of-the-barrier motion should be properly accounted for, see Ref. [27].

Numerical simulations To corroborate our analytical arguments, we have carried out an $a b$ initio simulation of the tunneling process in a highly charged ion in a laser field of relativistic intensities by solving the time-dependent Dirac Eq. (1) numerically [28]. Figure 4 shows the wave packet of the active electron at the moment when the laser electric field is at its maximum. Here one part of the wave packet is still bound or under the barrier while the other part is already in the continuum. The part under the barrier is not exactly symmetric to the laser polarization direction. There is a small drift motion into the laser propagation direction which is of the order $z \sim 1 / c$ in accordance with our estimation above.

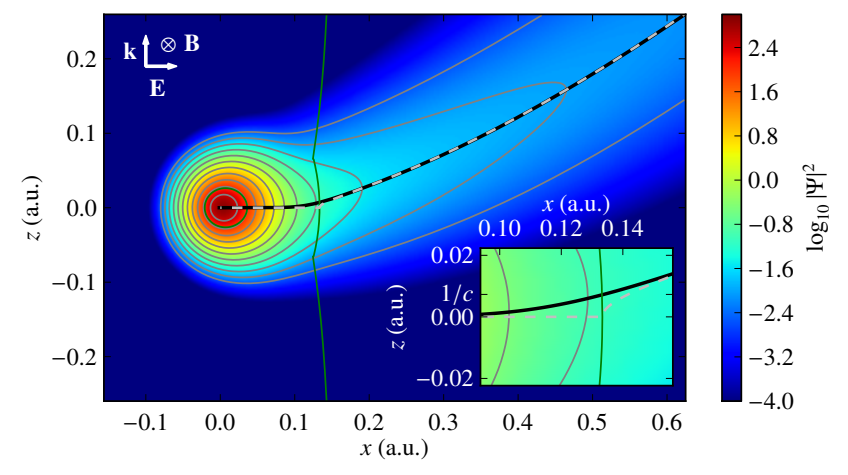

FIG. 4: (color online) Numerical simulation of the electron wave function in a soft-core potential via the Dirac equation. The density plot shows the electron density at the moment when maximal laser field strength is attained. The solid black line indicates the maximum of the density in the laser propagation direction while the dashed line corresponds to the most probable trajectory resulting from the quasiclassical description. Solid green lines correspond to the border of the classical forbidden region. White arrows and the cross indicate the directions of the laser's electromagnetic fields and its propagation direction. The inset shows a scale-up of the region close to the tunnel exit. The applied parameters are $I_{p} / c^{2}=0.25$, and $E_{0} / E_{a}=1 / 30$, and $\gamma=0.1$.

Conclusion Laser-driven relativistic tunneling dynamics was shown to be understood by a simple picture incorporating a scalar tunneling barrier and position-dependent energy levels. A spatial shift and a momentum shift along the laser propagation direction at the tunnel exit were identified as signatures of the relativistic dynamics through the tunneling barrier. The spatial drift is shown to be proportional to the EisenbudWigner-Smith time delay, while the momentum shift to the Keldysh time. While the spatial shift features intrinsic limitations for its measurement, the momentum shift is of the order of $10 \mathrm{a}$. u. for the applied parameters and within the reach of up-to-date experimental techniques [19].

We are grateful to C. Müller for valuable discussions at the onset of the project.

[1] V. Yanovsky, V. Chvykov, G. Kalinchenko, P. Rousseau, T. Planchon, T. Matsuoka, A. Maksimchuk, J. Nees, G. Cheriaux, G. Mourou, and K. Krushelnick, Opt. Express 16, 2109 (2008)

[2] H. G. Hetzheim and C. H. Keitel, Phys. Rev. Lett. 102, 083003 (2009), H. Bauke, H. G. Hetzheim, G. R. Mocken, M. Ruf, and C. H. Keitel, Phys. Rev. A 83, 063414 (2011)

[3] A. Di Piazza, C. Müller, K. Z. Hatsagortsyan, and C. H. Keitel, Rev. Mod. Phys. 84, 1177 (2012)

[4] H. R. Reiss, Phys. Rev. A 42, 1476 (1990), J. Opt. Soc. Am. B 7, 574 (1990)

[5] L. V. Keldysh, Zh. Eksp. Teor. Fiz. 47, 1945 (1964).

[6] F. H. M. Faisal, J. Phys. B: At. Mol. Phys. 6, L89 (1973)

[7] H. R. Reiss, Phys. Rev. A 22, 1786 (1980)

[8] V. S. Popov, V. D. Mur, and B. M. Karnakov, JETP Lett. 66 , 
229 (1997), V. D. Mur, B. M. Karnakov, and V. S. Popov, JETP Lett. 87, 433 (1998).

[9] A. M. Perelomov, V. S. Popov, and V. M. Terent'ev, Zh. Eksp. Teor. Fiz. 52, 514 (1967); M. V. Ammosov, N. B. Delone, and V. P. Krainov, 91, 2008 (1986).

[10] D. P. Crawford and H. R. Reiss, Phys. Rev. A 50, 1844 (1994) M. Klaiber, K. Z. Hatsagortsyan, and C. H. Keitel, Phys. Rev. A 75, 063413 (2007)

[11] N. Milosevic, V. P. Krainov, and T. Brabec, Phys. Rev. Lett. 89, 193001 (2002); J. Phys. B 35, 3515 (2002)

[12] S. Augst, D. Strickland, D. D. Meyerhofer, S. L. Chin, and J. H. Eberly, Phys. Rev. Lett. 63, 2212 (1989)

[13] Atomic units are used throughout the Letter unless stated otherwise.

[14] H. R. Reiss, Phys. Rev. Lett. 101, 043002 (2008)

[15] L. Eisenbud, Ph.D. thesis, Princeton University (1948); E. P. Wigner, Phys. Rev. 98, 145 (1955), F. T. Smith, Phys. Rev. 118, 349 (1960)

[16] L. A. MacColl, Phys. Rev. 40, 621 (1932)

[17] C. A. A. de Carvalho and H. M. Nussenzveig, Phys. Rep. 364, $83(2002)$

[18] A. I. Baz', Yad. Fiz. 4, 252 (1966); A. Peres, Am. J. Phys. 48, 552 (1980); P. C. W. Davies, Am. J. Phys. 73, 23 (2005) R. Landauer and T. Martin, Rev. Mod. Phys. 66, 217 (1994), D. Sokolovski, in Time in Quantum Mechanics, Lect. Notes Phys., Vol. 734, edited by J. G. Muga, R. Mayato, and Í. L. Egusquiza (Springer, Berlin, Heidelberg, 2008) pp. 195233; Y. Ban, E. Y. Sherman, J. G. Muga, and M. Büttiker, Phys. Rev. A 82, 062121 (2010), E. A. Galapon, Phys. Rev. Lett 108, 170402 (2012)

[19] P. Eckle, M. Smolarski, F. Schlup, J. Biegert, A. Staudte, M. Schöffler, Muller, R. Dörner, and U. Keller, Nat. Phys. 4, 565 (2008), P. Eckle, A. N. Pfeiffer, C. Cirelli, A. Staudte, R. Dörner, H. G. Muller, M. Büttiker, and U. Keller, Science 286, 1525 (2008), A. N. Pfeiffer, C. Cirelli, M. Smolarski, D. Dimitrovski, M. Abu-samha, L. B. Madsen, and U. Keller, Nat. Phys. 8, 76
(2012)

[20] H. R. Reiss, Phys. Rev. A 19, 1140 (1979)

[21] G. Grynberg, A. Aspect, and C. Fabre, Introduction to Quantum Optics: From the Semi-classical Approach to Quantized Light (Cambridge University Press, Cambridge, 2010).

[22] W. Becker, F. Grasbon, R. Kopold, D. Milošević, G. Paulus, and H. Walther, Adv. Atom. Mol. Opt. Phys. 48, 35 (2002) M. Klaiber, K. Z. Hatsagortsyan, and C. H. Keitel, Phys. Rev. A 73, 053411 (2006), ; M. Klaiber, E. Yakaboylu, and K. Z. Hatsagortsyan, Phys. Rev. A 87, 023418 (2013).

[23] D. M. Wolkow, Zeitschrift für Physik 94, 250 (1935).

[24] This is in contrast to situations with parameters close to over-thebarrier ionization resulting in a different momentum shift, see C. T. L. Smeenk, L. Arissian, B. Zhou, A. Mysyrowicz, D. M. Villeneuve, A. Staudte, and P. B. Corkum, Phys. Rev. Lett. 106, 193002 (2011), A. S. Titi and G. W. F. Drake, Phys. Rev. A 85, 041404 (2012)

[25] D. B. Milošević, S. Hu, and W. Becker, Phys. Rev. A 63, 011403 (2000), M. W. Walser, C. H. Keitel, A. Scrinzi, and T. Brabec, Phys. Rev. Lett. 85, 5082 (2000)

[26] E. Yakaboylu, M. Klaiber, H. Bauke, K. Z. Hatsagortsyan, and C. H. Keitel, In preparation.

[27] S. V. Popruzhenko, G. G. Paulus, and D. Bauer, Phys. Rev. A 77, 053409 (2008), T.-M. Yan and D. Bauer, Phys. Rev. A 86, 053403 (2012); J. C. Baggesen and L. B. Madsen, Phys. Rev. Lett. 104, 043602 (2010), A. S. Kheifets and I. A. Ivanov, Phys. Rev. Lett. 105, 233002 (2010), C.-H. Zhang and U. Thumm, Phys. Rev. A 82, 043405 (2010), S. Nagele, R. Pazourek, J. Feist, K. Doblhoff-Dier, C. Lemell, K. Tókési, and J. Burgdörfer, J. Phys. B: At. Mol. Opt. Phys. 44, 081001 (2011); M. Ivanov and O. Smirnova, Phys. Rev. Lett. 21, 213605 (2011); R. Pazourek, J. Feist, S. Nagele, and J. Burgdörfer, Phys. Rev. Lett. 108, 163001 (2012)

[28] H. Bauke and C. H. Keitel, Comput. Phys. Commun. 182, 2454 (2011) 